\title{
Implicancias de la glicemia de ingreso en la mortalidad intrahospitalaria y alejada según el método de reperfusión en pacientes con infarto agudo del miocardio y supradesnivel del segmento ST
}

\author{
DOUGLAS GREIG ${ }^{1}$, RAMÓN CORBALÁN ${ }^{1}$, \\ PABLO CASTRO ${ }^{1}$, PABLA CAMPOS ${ }^{2}$, RUBÉN LAMICH ${ }^{3}$, \\ PATRICIO YOVANINIZ ${ }^{3}$, CAROLINA NAZZAL ${ }^{4}$
}

\section{Admission hyperglycemia and mortality of ST segment elevation myocardial infarction}

Background: Hyperglycemia at admission has been associated to an adverse prognosis in patients with ST-segment elevation acute myocardial infarction (STEMI). However, its impact over the results of reperfusion therapies in patients with STEMI is still a matter of controversy. Aim: To determine the impact of admission hyperglycemia on hospital and long term mortality, according to the method of reperfusion utilized in patients with STEMI. Material and Methods: Prospective registry of 1,634 consecutive patients aged $60 \pm 12$ years ( $77 \%$ male), from 3 participating hospitals in the Chilean Registry of Myocardial Infarction (GEMI). We evaluated demographic, clinical and laboratory variables, reperfusion method used, hospital and long term mortality. The impact of hyperglycemia on hospital and long term mortality was evaluated by a logistic regression analysis and Cox risk, respectively, adjusted by Thrombolysis in Myocardial Infarction (TIMI) risk score. Results: Twenty four percent of patients were diabetics and in 45\%, the infarct was located on the anterior wall. The mean TIMI risk score was $3.2 \pm 2.4$. Hyperglycemia at entry was associated to a greater hospital and long term mortality, independently of the reperfusion strategy utilized. Primary angioplasty was associated to a greater benefit, compared to thrombolysis among hyperglycemic patients with an odds ratio: 2.9, 95\% confidence intervals: 1.0-8.0 and a hazard ratio of 2.9, 95\% confidence intervals: 1.44-5.88, independently of a previous history of diabetes mellitus and TIMI risk score. Conclusions: In patients with STEMI, admission hyperglycemia is associated with a worse prognosis which was significantly improved with primary angioplasty compared to thrombolysis, independently of the admission TIMI risk score.

(Rev Med Chile 2010; 138: 1109-1116).

Key words: Blood glucose; Myocardial infarction; Myocardial reperfusion.

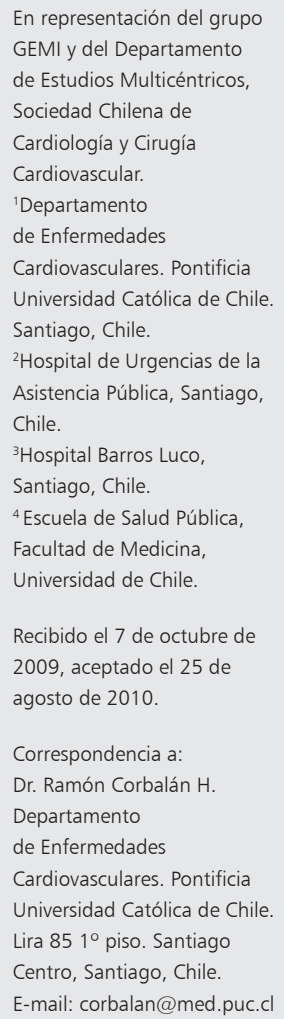<smiles>[2H]</smiles>
urante las últimas décadas, la introducción de terapias de reperfusión efectivas para el manejo del infarto con supradesnivel del segmento ST (IAM-SDST) como la trombolisis (TR) y la angioplastia primaria (APP), junto a terapias coadyuvantes, han modificado significativamente la expectativa de vida asociada a esta patología ${ }^{1}$. La presencia de hiperglicemia al ingreso, independiente del antecedente de diabetes mellitus (DM), ha sido identificada como un factor pronóstico adverso en IAM-SDST en múltiples estudios observacionales ${ }^{2-4}$. La información referente 
a los resultados de terapias de reperfusión en este subgrupo de pacientes es limitada, pero sugieren una mayor tasa de fracaso de la TR ${ }^{5,6}$. La APP en este grupo de pacientes ofrece ventajas teóricas, ya que se asocia a tasas mayores de reperfusión coronaria exitosa, mayor reducción de mortalidad y menor incidencia de complicaciones hemorrágicas ${ }^{1,7-9}$.

El objetivo de este estudio es investigar el impacto de la hiperglicemia al ingreso en la mortalidad intrahospitalaria y a largo plazo del IAMSDST según el método de reperfusión empleado.

\section{Método}

\section{Diseño y selección de los pacientes}

Estudio observacional. Se incluyeron 2.296 pacientes consecutivos desde enero de 2001 hasta marzo de 2006, ingresadoscon diagnóstico de IAM y SDST en 3 centros hospitalarios terciarios de la región metropolitana de Santiago de Chile participantes en el Registro Chileno del Infarto (GEMI) y que tratan más de 300 pacientes por año, 2 de ellos utilizan preferentemente TR y el otro APP. Los pacientes fueron incluidos si cumplían con los siguientes requisitos: 1 ) síntomas compatibles con IAM por al menos 30 minutos; 2) elevación persistente del segmento ST de al menos $0,1 \mathrm{mV}$ en dos derivadas contiguas en el ECG y 3 ) elevación de marcadores séricos de necrosis miocárdica mayor a 2 veces el rango normal (troponina $\mathrm{T}$ o I, CK total, CK-MB); 4) tratamiento con TR o APP dentro de las primeras 6 horas de evolución.

\section{Registro de los datos}

Los datos fueron ingresados en una ficha especialmente diseñada por una enfermera y/o médico responsable en cada institución. Se registraron datos demográficos, características clínicas del IAM (duración del dolor previo a la consulta, localización del IAM, KILLIP de ingreso, antecedentes coronarios y factores de riesgo entre otros), terapia de reperfusión utilizada y tiempo de inicio desde el momento de ingreso del paciente, necesidad de nueva coronariografía y/o cirugía de revascularización. Se consideró hiperglicemia de ingreso a la glicemia plasmática $\geq 140 \mathrm{mg} / \mathrm{dl}$, independiente del antecedente de diabetes mellitus. Este punto de corte se determinó mediante un modelo múltiple de riesgo proporcional de Cox y fue el mejor predictor de mortalidad alejada, según una comunicación anterior de nuestro grupo
(Greig D. et al. "Valor adicional de la glicemia de ingreso en la estratificación de riesgo en el infarto agudo al miocardio. Análisis en tres hospitales terciarios". XLIII Congreso Chileno de Cardiología y Cirugía Cardiovascular). Para la estratificación de riesgo del infarto se utilizó el puntaje de riesgo TIMI $^{10}$. Todos los análisis fueron realizados en forma centralizada por un observador externo a los centros hospitalarios involucrados. Se garantizó la privacidad de la información durante la ejecución del estudio.

\section{Seguimiento}

La mortalidad alejada y la causa de muerte se evaluaron mediante consulta a la base de datos de la Oficina de Registro Civil e Identificación al término del período de seguimiento. La mortalidad intrahospitalaria se evaluó en relación a los registros clínicos de los centros participantes. Para efectos del análisis se consideraron las muertes de origen cardiovascular.

\section{Análisis estadístico}

Los datos son presentados como promedio \pm desviación estándar. Los grupos fueron comparados mediante test $t$ de Student o test de chi cuadrado para variables continuas o categóricas según correspondía. La sobrevida se analizó mediante método de Kaplan-Meier; las curvas de sobrevida se compararon mediante el test de Log Rank (Figura 1). Para determinar el riesgo incremental de la glicemia en la mortalidad intrahospitalaria y alejada, la glicemia se categorizó en cuartiles en forma diferenciada para pacientes con y sin antecedentes de DM al ingreso, posteriormente, los modelos se ajustaron según las variables que fueron significativas en los modelos univariados, edad, sexo, infarto de pared anterior, KILLIP $>1$ y presión arterial sistólica (Figuras $2 \mathrm{a}$ y $2 \mathrm{~b}$ ). El rol pronóstico de la hiperglicemia al ingreso y el método de reperfusión, fue evaluado mediante un modelo de regresión logística múltiple, señalando los Odds ratio (OR) respectivos para mortalidad intrahospitalaria, mientras que para la evaluación del impacto en mortalidad alejada se empleó el modelo de riesgos proporcionales de Cox, señalando los Hazard ratio (HR) respectivos (Figuras $3 a$ y $3 b$ ). Ambos modelos fueron ajustados por puntaje de riesgo de TIMI. Un valor de $\mathrm{p} \leq 0,05$ de dos colas fue considerado significativo. Todos los análisis fueron realizados con SPSS 15.0. 


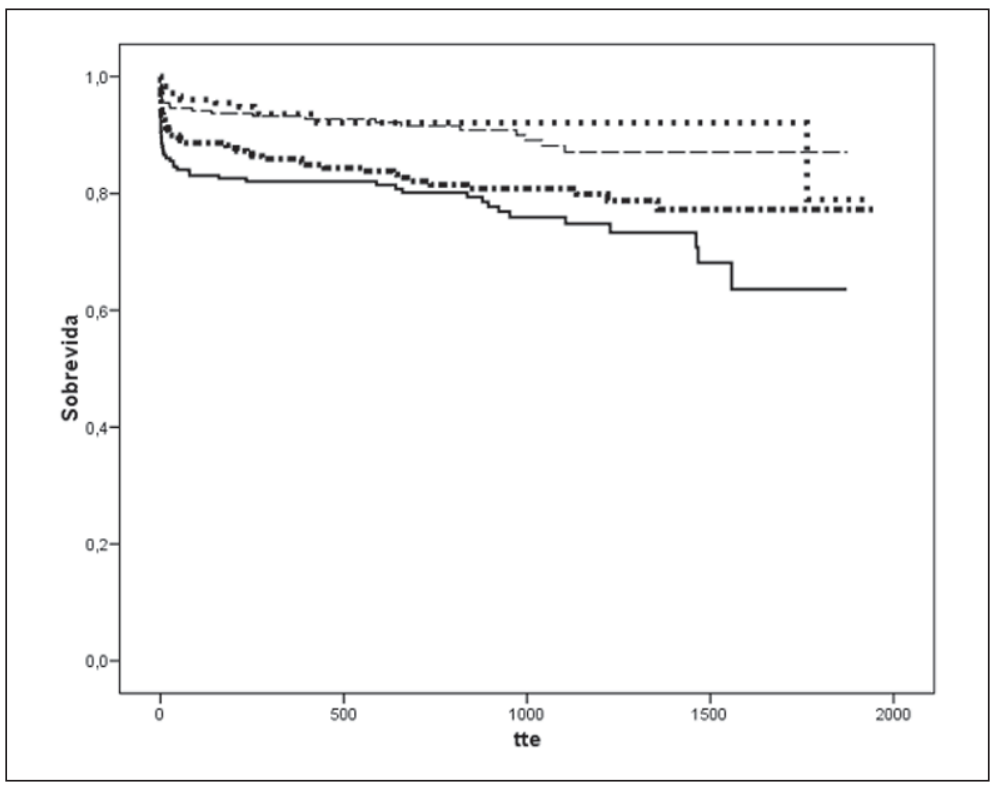

Figura 1. Curvas de sobrevida de acuerdo al cuartil de glicemia de ingreso. Línea punteado grueso representa al $1^{\circ}$ cuarti (glicemia menor a $117 \mathrm{mg} / \mathrm{dL}$ ). Línea continua oscura representa al $2^{\circ}$ cuartil (glicemia entre 117 y $138 \mathrm{mg} / \mathrm{dL}$ ). Línea continua oscura representa al $3^{\circ}$ cuartil (glicemia entre 139 y $199 \mathrm{mg} / \mathrm{dL}$ ). Línea punteado fino representa al $4^{\circ}$ cuartil (glicemia mayor a $200 \mathrm{mg} / \mathrm{dL}$ ).

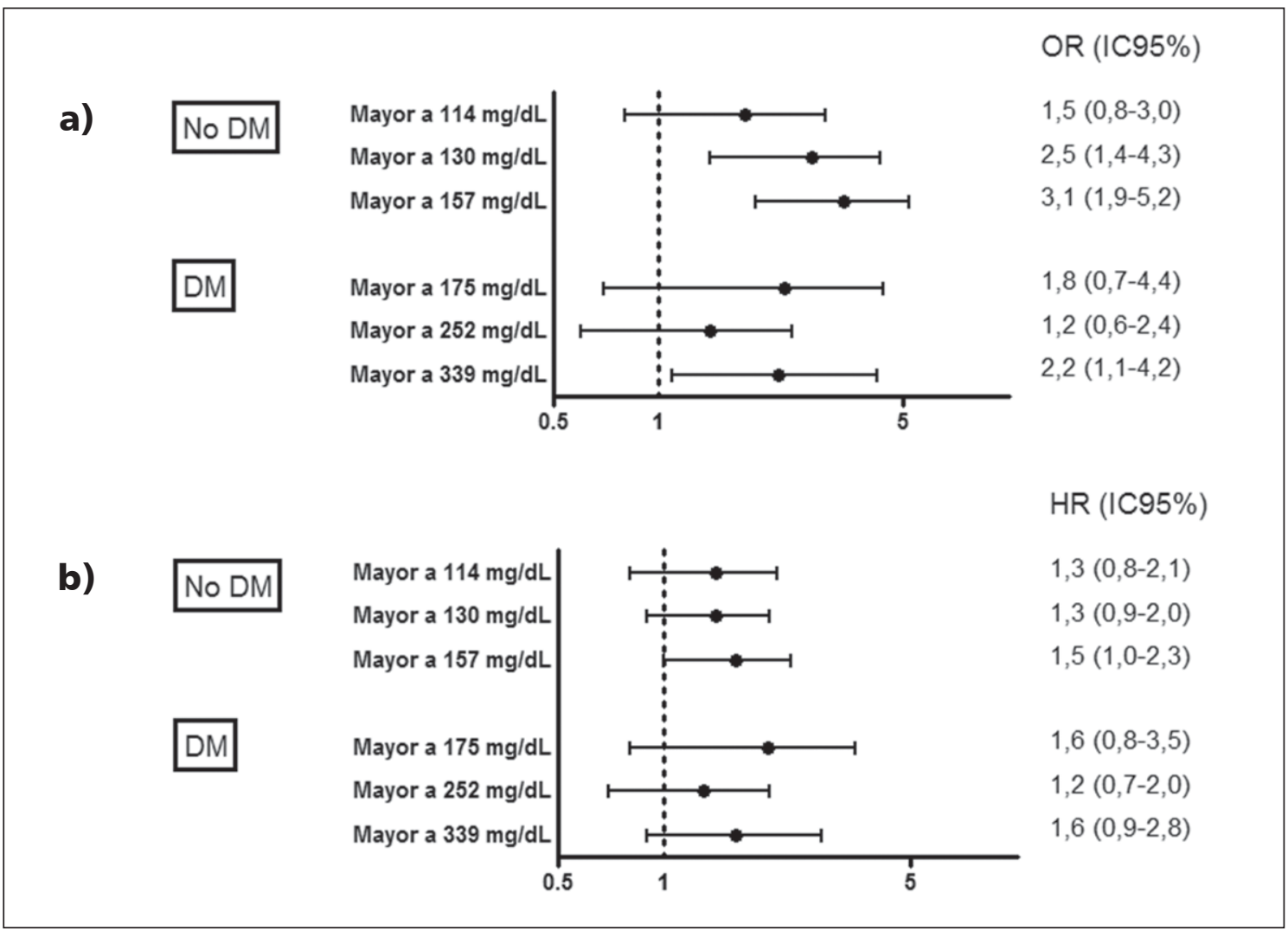

Figura 2. a) Mortalidad intrahospitalaria en pacientes con y sin diabetes mellitus según rangos de glicemia (cuartiles). b) Mortalidad alejada en pacientes con y sin diabetes mellitus según rangos de glicemia (cuartiles). 


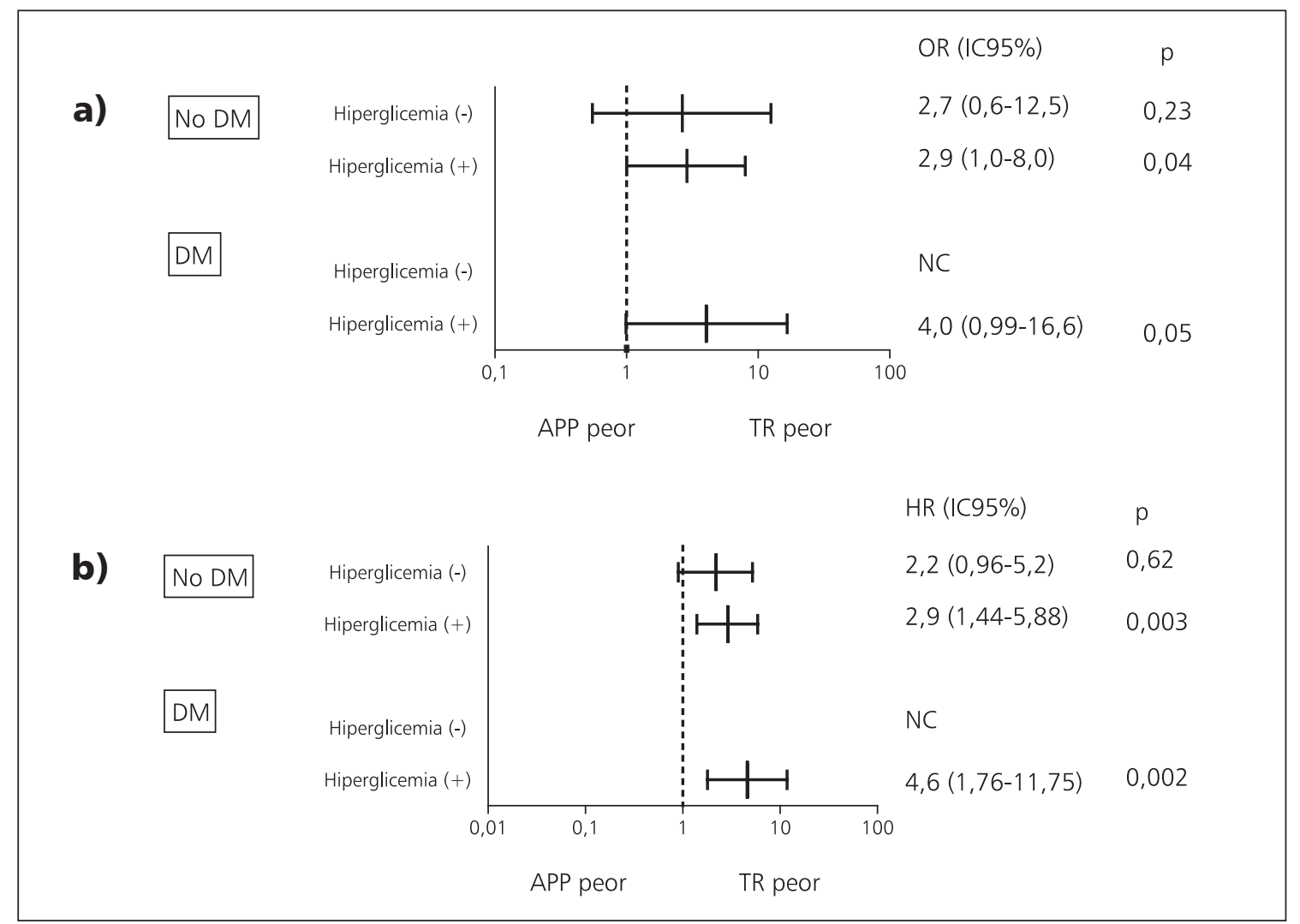

Figura 3. a) Mortalidad intrahospitalaria según método de reperfusión considerando glicemia de ingreso en pacientes diabéticos y no diabéticos b) Mortalidad alejada según método de reperfusión considerando glicemia de ingreso en pacientes diabéticos y no diabéticos (TR=trombolisis, APP=angioplastía primaria, NC: no calculable por ausencia de eventos).

\section{Resultados}

\section{Características basales de los pacientes}

De los 2.296 pacientes incluidos en el registro, $1.634(71 \%)$ correspondieron a IAM con SDST. De éstos, $967(59,2 \%)$ fueron tratados con terapias de reperfusión, $72 \%$ con TR (90\% con estreptoquinasa y $10 \%$ con trombolíticos específicos) y $28 \%$ con APP, lo que constituye la muestra de nuestro estudio. Las principales causas para no utilizar terapias de reperfusión fueron: ingreso tardío (46\%), ausencia de angina al ingreso (16\%) y contraindicaciones formales al uso de trombolíticos (10\%).

El promedio de edad fue de $60 \pm 12$ años, $77 \%$ fueron de sexo masculino, $24 \%$ diabéticos y $55 \%$ hipertensos. El promedio de la glicemia de ingreso fue de $264 \pm 114 \mathrm{mg} / \mathrm{dL}$ en pacientes con DM y 144 $\pm 50 \mathrm{mg} / \mathrm{dl}$ en pacientes sin historia de DM. Las características basales de los 967 pacientes según la presencia o ausencia de hiperglicemia de ingreso, se muestran en la Tabla 1.

Se observa que los pacientes con hiperglicemia de ingreso fueron en mayor proporción de sexo femenino, tenían mayor edad y mayor prevalencia de HTA. Además, tenían infartos clínicamente más graves, caracterizados por un mayor puntaje de riesgo TIMI e insuficiencia cardíaca (KILLIP $\geq 1$ ) al ingreso.

El seguimiento se obtuvo en el 100\%. La causa de muerte fue identificable en $98 \%$ de los casos. El promedio de seguimiento fue de 859 días (rango 30-1944). En el grupo total, la mortalidad intrahospitalaria en pacientes tratados con TR y APP fue de 10,9 vs $5,6 \%(\mathrm{p}<0,01)$ y la mortalidad alejada fue de 20,4 vs $9,7 \%(\mathrm{p}<0,01)$, respectivamente. 
Tabla 1. Características basales de los pacientes según la presencia o no de hiperglicemia al ingreso

\begin{tabular}{|c|c|c|c|}
\hline$n=967$ & $\begin{array}{l}\text { HG (+) } \\
(54 \%)\end{array}$ & $\begin{array}{l}\text { HG (-) } \\
(46 \%)\end{array}$ & Valor-p \\
\hline Edad (promedio $\pm \mathrm{DE}$ ) & $63 \pm 12$ & $59 \pm 13$ & $<0,01$ \\
\hline Sexo masculino (\%) & 75 & 80 & 0,07 \\
\hline HTA (\%) & 54 & 47 & 0,05 \\
\hline Diabetes mellitus (\%) & 37 & 5 & $<0,01$ \\
\hline Dislipidemia (\%) & 30 & 35 & 0,07 \\
\hline Tabaquismo (\%) & 82 & 82 & 1,0 \\
\hline IAM previo (\%) & 12 & 9 & 0,22 \\
\hline $\begin{array}{l}\text { Revascularización previa (\%) } \\
\text { Angioplastía } \\
\text { CRM }\end{array}$ & $\begin{array}{l}3 \\
3\end{array}$ & $\begin{array}{l}3 \\
2\end{array}$ & $\begin{array}{l}1,0 \\
0,38\end{array}$ \\
\hline \multicolumn{4}{|l|}{ Características del infarto } \\
\hline KILLIP > $1(\%)$ & 24 & 13 & $<0,001$ \\
\hline Puntaje TIMI 33 (\%) & 63 & 49 & $<0,001$ \\
\hline Tiempo a tratamiento $(\min \pm \mathrm{DE})$ & $47 \pm 48$ & $49 \pm 38$ & 0,58 \\
\hline Localización pared anterior (\%) & 45 & 45 & 1,0 \\
\hline Angioplastía primaria (\%) & 31 & 34 & 0,45 \\
\hline
\end{tabular}

HG (+): hiperglicemia de ingreso, HG (-): sin hiperglicemia de ingreso, HTA=hipertensión arterial, IAM=infarto agudo del miocardio $C R M=$ cirugía de revascularización miocárdica.

Tabla 2. Mortalidad intrahospitalaria y alejada no ajustada según método de reperfusión y presencia o ausencia de hiperglicemia, en pacientes con y sin diabetes mellitus

\begin{tabular}{|lccccccc|}
\hline & $\begin{array}{c}\text { Mortalidad intrahospitalaria } \\
\text { (\%) }\end{array}$ & \multicolumn{2}{c}{} & \multicolumn{3}{c}{$\begin{array}{c}\text { Mortalidad al año } \\
\text { (\%) }\end{array}$} \\
Pacientes & APP & TR & p & APP & TR & p \\
\hline DM (-) HG (+) & 9,1 & 11,2 & 0,68 & 8,0 & 15,7 & 0,09 \\
DM (-) HG (-) & 1,6 & 4,1 & 0,35 & 4,0 & 7,8 & 0,19 \\
\hline DM (+) HG (+) & 7,1 & 20,9 & 0,06 & 7,1 & 22,5 & 0,04 \\
\hline
\end{tabular}

HG (+): hiperglicemia de ingreso, HG (-): sin hiperglicemia de ingreso. DM=Diabetes mellitus.

Efectos de la glicemia de ingreso y método de reperfusión en la mortalidad

La presencia de hiperglicemia al ingreso se asoció a una mayor mortalidad intrahospitalaria y alejada. La Figura 1 muestra las curvas de sobrevida según cuartil de glicemia de ingreso para el grupo total ( $\log$ Rank test, $\mathrm{p}<0,01)$. Se observa en esta figura que los cuartiles $3^{\circ}$ y $4^{\circ}(140-199$ y sobre $200 \mathrm{mg} / \mathrm{dl}$, respectivamente) se asociaron a una menor sobrevida en el seguimiento alejado.
Del mismo modo, la presencia de hiperglicemia de ingreso se tradujo en una mayor mortalidad intrahospitalaria y al año (Tabla 2).

La Figura 2 muestra la relación de la glicemia de ingreso según cuartiles y mortalidad intrahospitalaria (Figura 2a) y alejada (Figura 2b), para pacientes diabéticos y no diabéticos. Según se observa en estos modelos ajustados por características del infarto, edad y sexo, la glicemia de ingreso constituye un factor de riesgo adverso e 
incremental, principalmente en los pacientes no diabéticos y particularmente, al considerar la mortalidad alejada. En ambos modelos los valores de glicemia que confieren un mayor riesgo son más elevados en los pacientes diabéticos comparados a los no diabéticos.

La Figura 3 muestra la mortalidad, intrahospitalaria (Figura 3a) y alejada (Figura 3b) para pacientes con y sin hiperglicemia de ingreso y método de reperfusión empleado, en pacientes con y sin antecedentes de diabetes mellitus. Al igual que lo señalado en la tabla 2, la mortalidad alejada e intrahospitalaria es mayor en pacientes con hiperglicemia al ingreso. Más aún, la APP ofreció un mayor beneficio en relación a TR en ambos grupos, sin embargo, este efecto es significativamente superior en pacientes hiperglicémicos, independiente de la presencia de diabetes mellitus.

\section{Discusión}

El presente estudio confirma que en pacientes con IAM-SDST la hiperglicemia al ingreso es un factor de riesgo para mortalidad intrahospitalaria y alejada. El beneficio proporcional de APP sobre TR, observado en el grupo general, es mayor en pacientes con hiperglicemia al ingreso, independiente de la gravedad del cuadro clínico estimado por puntaje de riesgo de TIMI.

La elevación transitoria de los niveles de glicemia es común en las primeras horas del IAM ${ }^{6}$. Numerosos estudios observacionales han asociado la presencia de hiperglicemia al ingreso con un peor pronóstico del IAM, relacionándola con un mayor tamaño del IAM y compromiso de la función ventricular ${ }^{4,11,12}$. El mecanismo fisiopatológico del daño provocado por hiperglicemia en el IAM no está claro, postulándose como responsables al aumento de marcadores inflamatorios y activación inmune ${ }^{13,14}$, inhibición de la expresión de iNOS mediada por isquemia ${ }^{15} \mathrm{y}$ disminución del pre-acondicionamiento isquémico mediada por cierre de canales $\mathrm{K}_{\text {ATP }}^{16}$. Otro mecanismo potencial de daño por hiperglicemia se asocia al desarrollo de disfunción endotelial e inhibición de la angiogénesis ${ }^{17,18}$. Estudios experimentales sugieren que la hiperglicemia potencia la adhesión plaquetaria, aumenta las moléculas de adhesión solubles y disminuye la biodisponibilidad de óxido nítrico, lo que puede llevar a alteraciones del flujo coro- nario $^{7,19}$. Del mismo modo, el estudio CLARITY TIMI 28, mostró que en pacientes con infarto tratados con trombolisis, la presencia de hiperglicemia al ingreso se asoció a mayor fracaso de arteria abierta (Flujo TIMI 2/3), independiente del uso de clopidogrel ${ }^{20}$. Estos mecanismos podrían contribuir a explicar la mayor tasa de fracaso de reperfusión observada en diabéticos sometidos a trombolisis 6 .

Observaciones recientes muestran que la APP tiene un mayor impacto en reducir la mortalidad en comparación a la TR en pacientes diabéticos ${ }^{21}$. Sin embargo, en el grupo de pacientes con hiperglicemia al ingreso el impacto potencial a largo plazo de terapias como la APP aun no está del todo aclarado $^{22-24}$. Nuestros resultados son diferentes a los reportados por Ishihara et al ${ }^{8}$, quien encontró que el mayor beneficio de la APP en pacientes con hiperglicemia se limitaba a la mortalidad a 30 días, a diferencia de los pacientes con antecedente de DM, en los cuales el beneficio de la APP se mantiene en el tiempo. En nuestro estudio, los beneficios de la APP sobre la TR en pacientes con hiperglicemia de ingreso se mantienen en el seguimiento alejado. Este efecto se mantuvo luego del ajuste por puntaje TIMI, lo que parece contradecir la hipótesis de que la hiperglicemia de ingreso constituye solamente un marcador de la severidad del infarto y no un factor pronóstico independiente en el IAM-SDST ${ }^{5,25}$.

Es interesante resaltar que los niveles de glicemia de ingreso que confieren un riesgo incremental de mortalidad son diferentes en pacientes diabéticos y no diabéticos. Al igual que en otros reportes $3,7,12,26,27$ los niveles de glicemia asociados a mortalidad en pacientes diabéticos son proporcionalmente superiores, y el impacto de la hiperglicemia como factor de riesgo independiente, es menor en este subgrupo. Esto puede obedecer, al menos en parte, a las características clínicas y demográficas de los pacientes diabéticos. En efecto, en nuestro grupo, los pacientes diabéticos tuvieron infartos clínicamente más graves, menor tasa de reperfusión exitosa y eran de edad más avanzada que en el grupo no diabético (datos no mostrados). Estos factores podrían contribuir al pronóstico adverso asociado a diabetes mellitus independientemente del valor de glicemia de ingreso. Por otro lado, nuestros datos son concordantes con los publicados con los de Petursson et al, quienes describieron que en pacientes con hiperglicemia 
Glicemia al ingreso, reperfusión y mortalidad por infarto cardíaco - D. Greig et al

de ingreso sin el antecedente de diabetes mellitus tenían una mortalidad mayor comparada a los pacientes con hiperglicemia y el antecedente de diabetes $^{28}$. En nuestro trabajo, esta observación se mantiene, además, en el seguimiento a largo plazo.

La ausencia de determinaciones seriadas de glicemia constituye una de las limitaciones de nuestro estudio ya que impide evaluar qué porcentaje de los pacientes con hiperglicemia de ingreso correspondían a diabetes mellitus no diagnostica$\mathrm{da}^{29}$. Además, el momento en que se determina la glicemia parece ser determinante en el establecer el pronóstico de sobrevida. De este modo, Nicolau et al, ha mostrado que la persistencia de hiperglicemia a las 24 horas de evolución se asocia a un peor pronóstico alejado ${ }^{30}$, mientras que Vivas et al, reportó que la primera glicemia en ayuna parece ser un mejor predictor de mortalidad, comparado a la glicemia de ingreso ${ }^{31}$.

En conclusión, en pacientes con IAM-SDST, la presencia de hiperglicemia se asocia a una mayor mortalidad intrahospitalaria y alejada. El beneficio proporcional de AP vs. TR se incrementa en relación a hiperglicemia al ingreso en ambos grupos, independiente del antecedente de DM. Estos hallazgos confirman que el beneficio de la APP con respecto de la TR es más significativo en la medida que aumenta el perfil de riesgo de los pacientes. Los posibles alcances terapéuticos de estas observaciones deben ser evaluados en futuros estudios prospectivos.

Nota del Editor: los autores indican que "este trabajo se escribió en el contexto del registro GEMI, el cual se encuentra avalado por el Comité de Ética de la Sociedad Chilena de Cardiología”.

\section{Referencias}

1. Keeley EC, Boura JA, Grines CL. Primary angioplasty versus intravenous thrombolytic therapy for acute myocardial infarction: a quantitative review of 23 randomised trials. Lancet 2003; 361: 13-20.

2. Norhammar A, Tenerz A, Nilsson G, Hamsten A, Efendic $\mathrm{S}$, Ryden L, et al. Glucose metabolism in patients with acute myocardial infarction and no previous diagnosis of diabetes mellitus: a prospective study. Lancet 2002; 359: 2140-4.

3. Timmer JR, van dH, I, Ottervanger JP, Henriques JP, Hoorntje JC, de Boer MJ, et al. Prognostic value of admission glucose in non-diabetic patients with myocardial infarction. Am Heart J 2004; 148: 399-404.

4. Schiele F, Scotes-Genon V, Seronde MF, Blonde MC, Legalery P, Meneveau N, et al. Predictive value of admission hyperglycaemia on mortality in patients with acute myocardial infarction. Diabet Med 2006; 23: 1370-6.

5. Wahab NN, Cowden EA, Pearce NJ, Gardner MJ, Merry $\mathrm{H}$, Cox JL. Is blood glucose an independent predictor of mortality in acute myocardial infarction in the thrombolytic era? J Am Coll Cardiol 2002; 40: 1748-54.

6. Oswald GA, Corcoran S, Yudkin JS. Prevalence and risks of hyperglycaemia and undiagnosed diabetes in patients with acute myocardial infarction. Lancet 1984; 1: 1264-7.

7. Timmer JR, Ottervanger JP, de Boer MJ, Dambrink JH, Hoorntje JC, Gosselink AT, et al. Hyperglycemia is an important predictor of impaired coronary flow before reperfusion therapy in ST-segment elevation myocardial infarction. J Am Coll Cardiol 2005; 45: 999-1002.

8. Ishihara M, Inoue I, Kawagoe T, Shimatani Y, Kurisu S, Nishioka K, et al. Impact of acute hyperglycemia on left ventricular function after reperfusion therapy in patients with a first anterior wall acute myocardial infarction. Am Heart J 2003; 146: 674-8.

9. Ishihara M, Kagawa E, Inoue I, Kawagoe T, Shimatani Y, Kurisu S, et al. Impact of admission hyperglycemia and diabetes mellitus on short- and long-term mortality after acute myocardial infarction in the coronary intervention era. Am J Cardiol 2007; 99: 1674-9.

10. Morrow DA, Antman EM, Charlesworth A, Cairns R, Murphy SA, de Lemos JA, et al. TIMI risk score for STelevation myocardial infarction: A convenient, bedside, clinical score for risk assessment at presentation: An intravenous nPA for treatment of infarcting myocardium early II trial substudy. Circulation 2000; 102: 2031-7.

11. Ishihara M, Kojima S, Sakamoto T, Asada Y, Tei C, Kimura $\mathrm{K}$, et al. Acute hyperglycemia is associated with adverse outcome after acute myocardial infarction in the coronary intervention era. Am Heart J 2005; 150: 814-20.

12. Stranders I, Diamant M, van Gelder RE, Spruijt HJ, Twisk JW, Heine RJ, et al. Admission blood glucose level as risk indicator of death after myocardial infarction in patients with and without diabetes mellitus. Arch Intern Med 2004; 164: 982-8.

13. Mahon NG, McKenna CJ, Codd MB, O'Rorke C, McCann HA, Sugrue DD. Gender differences in the management and outcome of acute myocardial infarction in unselected patients in the thrombolytic era. Am J Cardiol 2000; 85: 921-6.

14. Webster KA. Stress hyperglycemia and enhanced sensitivity to myocardial infarction. Curr Hypertens Rep 2008; 10: 78-84. 
15. Marfella R, Di FC, Esposito K, Nappo F, Piegari E, Cuzzocrea $\mathrm{S}$, et al. Absence of inducible nitric oxide synthase reduces myocardial damage during ischemia reperfusion in streptozotocin-induced hyperglycemic mice. Diabetes 2004; 53: 454-62.

16. Ishihara M, Inoue I, Kawagoe T, Shimatani Y, Kurisu S, Nishioka K, et al. Effect of acute hyperglycemia on the ischemic preconditioning effect of prodromal angina pectoris in patients with a first anterior wall acute myocardial infarction. Am J Cardiol 2003; 92: 288-91.

17. Tuo QH, Zeng H, Stinnett A, Yu H, Aschner JL, Liao DF, et al. Critical role of angiopoietins/Tie-2 in hyperglycemic exacerbation of myocardial infarction and impaired angiogenesis. Am J Physiol Heart Circ Physiol 2008; 294: H2547-57.

18. Venkatachalam K, Mummidi S, Cortez DM, Prabhu SD, Valente AJ, Chandrasekar B. Resveratrol inhibits high glucose-induced PI3K/Akt/ERK-dependent interleukin-17 expression in primary mouse cardiac fibroblasts. Am J Physiol Heart Circ Physiol 2008; 294: H2078-87.

19. Iwakura K, Ito H, Ikushima M, Kawano S, Okamura A, Asano K, et al. Association between hyperglycemia and the no-reflow phenomenon in patients with acute myocardial infarction. J Am Coll Cardiol 2003; 41: 1-7.

20. Pinto DS, Kirtane AJ, Pride YB, Murphy SA, Sabatine MS, Cannon CP, et al. Association of blood glucose with angiographic and clinical outcomes among patients with ST-segment elevation myocardial infarction (from the CLARITY-TIMI-28 study). Am J Cardiol 2008; 101: 303-7.

21. Timmer JR, Ottervanger JP, de Boer MJ, Boersma E, Grines CL, Westerhout CM, et al. Primary percutaneous coronary intervention compared with fibrinolysis for myocardial infarction in diabetes mellitus: results from the Primary Coronary Angioplasty vs Thrombolysis-2 trial. Arch Intern Med.\} 2007; 167: 1353-5\}9.

22. Lavi S, Kapeliovich M, Gruberg L, Roguin A, Boulos M, Grenadier E, et al. Hyperglycemia during acute myocardial infarction in patients who are treated by primary percutaneous coronary intervention: impact on longterm prognosis. Int J Cardiol 2008; 123: 117-22.

23. Gasior M, Stasik-Pres G, Pres D, Lech P, Gierlotka M,
Lekston A, et al. Relationship between blood glucose on admission and prognosis in patients with acute myocardial infarction treated with percutaneous coronary intervention. Kardiol Pol 2007; 65: 1031-8.

24. Straumann E, Kurz DJ, Muntwyler J, Stettler I, Furrer M, Naegeli B, et al. Admission glucose concentrations independently predict early and late mortality in patients with acute myocardial infarction treated by primary or rescue percutaneous coronary intervention. Am Heart J 2005; 150: 1000-6.

25. Capes SE, Hunt D, Malmberg K, Gerstein HC. Stress hyperglycaemia and increased risk of death after myocardial infarction in patients with and without diabetes: a systematic overview. Lancet 2000; 355: 773-8.

26. Timmer JR, van dH, I, Ottervanger JP, Henriques JP, Hoorntje JC, de Boer MJ, et al. Prognostic value of admission glucose in non-diabetic patients with myocardial infarction. Am Heart J 2004; 148: 399-404.

27. Kosiborod M, Rathore SS, Inzucchi SE, Masoudi FA, Wang Y, Havranek EP, et al. Admission glucose and mortality in elderly patients hospitalized with acute myocardial infarction: implications for patients with and without recognized diabetes. Circulation 2005; 111 : 3078-86.

28. Petursson P, Herlitz J, Caidahl K, Gudbjornsdottir S, Karlsson T, Perers E, et al. Admission glycaemia and outcome after acute coronary syndrome. Int J Cardiol 2007; 116: 315-20.

29. Ishihara M, Inoue I, Kawagoe T, Shimatani Y, Kurisu $\mathrm{S}$, Hata $\mathrm{T}$, et al. Is admission hyperglycaemia in nondiabetic patients with acute myocardial infarction a surrogate for previously undiagnosed abnormal glucose tolerance? Eur Heart J 2006; 27: 2413-9.

30. Goyal A, Mahaffey KW, Garg J, Nicolau JC, Hochman JS, Weaver WD, et al. Prognostic significance of the change in glucose level in the first $24 \mathrm{~h}$ after acute myocardial infarction: results from the CARDINAL study. Eur Heart J 2006; 27: 1289-97.

31. Vivas D, García-Rubira JC, González-Ferrer JJ, NúñezGil I, del PN, Fernández-Ortiz A, et al. [Prognostic value of first fasting glucose measurement compared with admission glucose level in patients with acute coronary syndrome]. Rev Esp Cardiol 2008; 61: 458-64. 